\title{
Factors associated with psychological distress of Public Health Nurse in Kagawa prefecture, Japan: A pilot study
}

\author{
Noriko Sakano $^{1 *}$, Takeshi Suzue ${ }^{2}$, Nobuyuki Miyatake ${ }^{1}$, Yoshikazu Miyamae ${ }^{3}$, Taichi Nagatomi ${ }^{4}$, \\ Takeshi Yoda ${ }^{2}$, Akira Yoshioka ${ }^{2}$, Wataru Shiraki ${ }^{5}$, Tomohiro Hirao ${ }^{2}$
}

\footnotetext{
${ }^{1}$ Department of Hygiene, Faculty of Medicine, Kagawa University, Kagawa, Japan

${ }^{2}$ Department of Public Health, Faculty of Medicine, Kagawa University, Kagawa, Japan

${ }^{3}$ Center for Educational Research and Teacher Development, Faculty of Education, Kagawa University, Kagawa, Japan

${ }^{4}$ Institute of Research Partnership Center for Social Collaboration and Intellectual Property, Kagawa University, Kagawa, Japan

${ }^{5}$ Department of Reliability-Based Information Systems Engineering, Faculty of Engineering, Kagawa University, Kagawa, Japan

Email: snoriko@med.kagawa-u.ac.jp
}

Received 14 September 2011; revised 16 December 2011; accepted 18 January 2012

\begin{abstract}
We evaluated the psychological distress using a scale of the K6, the 6-item scale of psychological distress on public health nurse in Kagawa prefecture, Japan. We sent the questionnaire to all public health nurses $(n=$ 419) in Kagawa prefecture. Then, a total of 256 public health nurses (1 man and 255 women), had completely answered the questionnaires, were analyzed in a cross-sectional investigation study. The association between psychological distress and considerable factors were evaluated by using the $\mathrm{K6}$, with psychological distress defined as $\mathbf{1 3}$ or more points out of a total of 24 points. A total of 15 public health nurses (5.9\%) were defined as psychological distress. Clear relationships between the $\mathrm{K6}$ score and age, experience duration as public health nurse were not noted. The K6 score in subjects with anxiety of preventing suicide was significantly higher than that without. In addition, the K6 score in subjects who felt their own mental status was excellent was also lower than that in subjects without that. In conclusion, some factors might be associated with psychological distress, as assessed by the K6, in public health nurse in Kagawa prefecture, Japan.
\end{abstract}

Keywords: Psychological Distress; K6; Public Health Nurse; Kagawa; Japan

\section{INTRODUCTION}

About $14 \%$ of the global disease burden has been attributed to mental illness, mostly due to the chronically disabling nature of depression and other common mental disorders [1]. Mental health has become public health challenge in Japan. For example, over 30 thousand sub-

\footnotetext{
${ }^{*}$ Corresponding author.
}

jects suicide in a year for over 10 years [2].

Recently, the demand for nurses has been increasing in various areas because of a medical reform bill and government amendments to the medical service payment structure in Japan. In addition, public health nurse, who works for community and company for public health in Japan, has become also more required. In some literatures, the psychological distress of nurses in hospitals has reported [3-7]. However, the evaluation of psychological distress of public health nurses in Japan still remains to be investigated.

Therefore, we evaluated the psychological distress of public health nurses by using the K6, the scale of psychological distress, in Kagawa prefecture, Japan and its relation to considerable factors in this cross-sectional investigation study.

\section{SUBJECTS AND METHODS}

\subsection{Subjects}

We used data of 271 public health nurses among 419 public health nurses in Kagawa prefecture, Japan, who met the following criteria; 1) they had completely answered the questionnaires and 2) they provided informed consent. Therefore, we analyzed 256 (61.1\%) public health nurses ( 1 man and 255 women), aged $42.5 \pm 11.2$ years. Ethical approval for the study was obtained from the Ethical Committee of Faculty of Medicine, Kagawa University, Japan.

\subsection{Psychological Distress}

The K6 was used as and indicator of psychological distress $[8,9]$. The 6 questions were as follows: "Over the last month, how often did you feel: 1) nervous; 2) hopeless; 3) restless or fidgety; 4) so sad that nothing could cheer you up; 5) that every thing was an effort; 6) worth- 
less?” (Table 1). Participants were asked to respond by choosing "all of the time" (4 points), "most of the time" (3 points), "some of the time" (2 points), "a little of the time" ( 1 point), and "none of the time" ( 0 points). Therefore, total point score ranged from 0 to 24 . The K6 has been developed using modern psychometric theory and has been shown to be superior to some existing scales in brevity and psychometric properties [8-10]. The Japanese version of the K6 has been recently developed, using the standard back-translation method, and has been validated [11]. As suggested by Kessler et al. [10], we classified participants with scores of 13 points or more as having psychological distress.

\subsection{Measurement of Variables}

The questionnaires for public health nurses consisted of the following details in sequence: age, sex, experience duration as public health nurses. In addition, we evaluated the consciousness of suicide, anxiety of preventing suicide and cooperation with specialist in their daily work.

\subsection{Statistical Analysis}

Data are expressed as mean \pm standard deviation (SD) values. A statistical analysis was performed using ANOVA and Scheffe's F test. Simple correlation analysis was performed as well to test for the significance of the linear relationship among continuous variables, where $p<0.05$ was considered to be statistically significant.

\section{RESULTS}

A total of 15 public health nurses (5.9\%) was defined as psychological distress. We evaluated the relationship between the K6 score and age (Figure 1), experience duration as public health nurse (Figure 2). Clear relationships between the K6 score and age $(\mathrm{r}=-0.175, p=$ 0.005 ), and between the $\mathrm{K} 6$ score and experience duration as public health nurse $(r=-0.133, p=0.034)$ were not noted in this study.

Table 1. The K6 included 6 questions was a scale of psychological distress.

Q: During the past 30 days, about how often did you feel

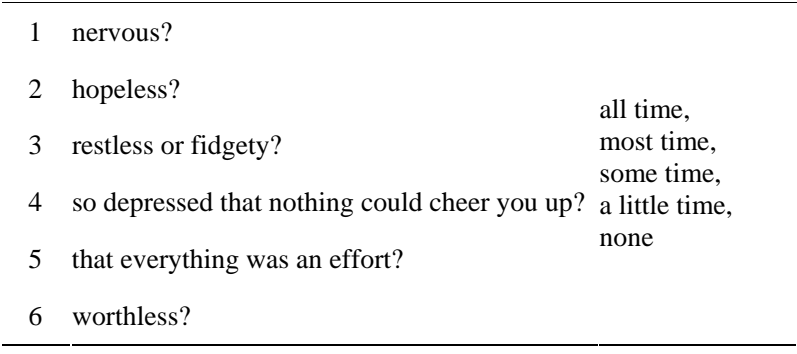

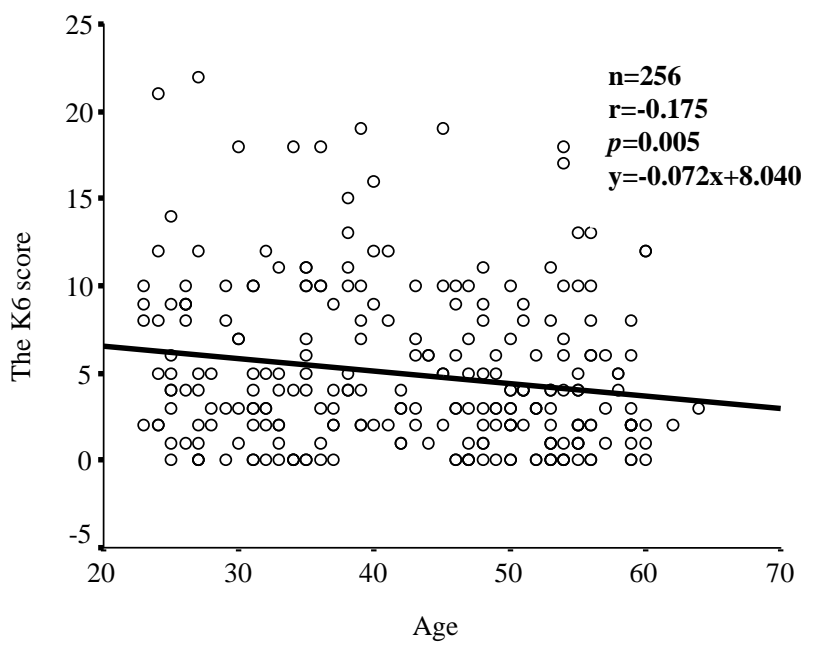

Figure 1. Relationship between the K6 score and age in public health nurse in Kagawa prefecture, Japan.

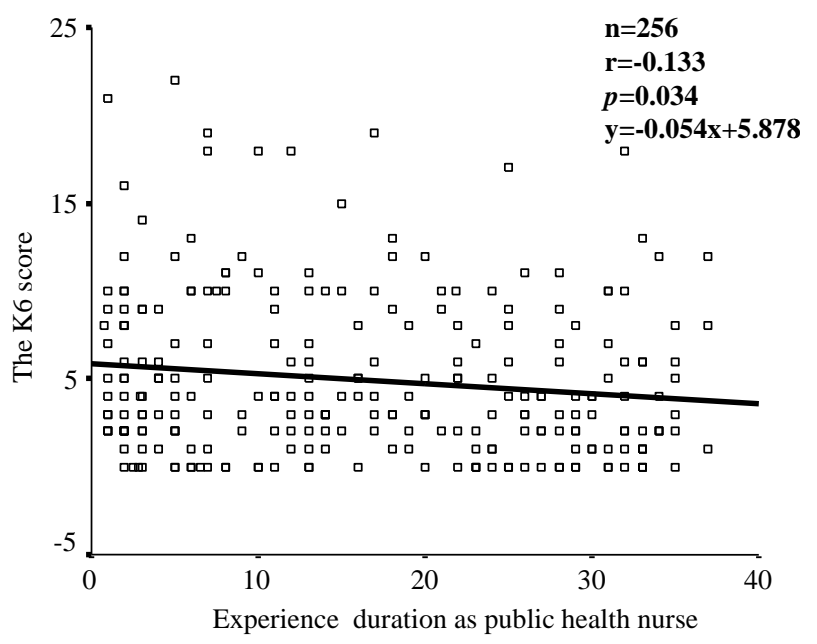

Figure 2. Relationship between the K6 score and experience duration as public health nurse in Kagawa prefecture, Japan.

In addition, we evaluated the factors i.e. consciousness of suicide, anxiety of preventing suicide and cooperation with specialist (Table 2). The K6 score in subjects with anxiety of preventing suicide was significantly higher than that without $(p=0.002)$. The K6 score in subjects without cooperation with specialist was also higher than that in subjects with that, but not at a significant level ( $p$ $=0.239$ ). However, significant differences were not noted between subjects with and without consciousness of suicide $(p=0.772)$.

Finally, the K6 score in subjects who feel their own mental status was excellent was also lower than that in subjects feeling bad or poor, by one way ANOVA (Figure 3).

\section{DISCUSSION}

We firstly evaluated the psychological distress using the 
Table 2. Relation between the K6 score and considerable factors in public health nurse.

\begin{tabular}{|c|c|c|c|c|c|}
\hline & Most of the time & Some of the time & A little of the time & None of the time & $p$-value \\
\hline \multicolumn{6}{|l|}{ Consciousness of suiside } \\
\hline Number of subjects & 21 & 161 & 67 & 7 & \\
\hline The K6 socre & $4.1 \pm 3.5$ & $5.2 \pm 4.6$ & $4.8 \pm 4.6$ & $4.4 \pm 7.0$ & 0.772 \\
\hline \multicolumn{6}{|l|}{ Anxiety of preventing suicide } \\
\hline Number of subjects & 168 & 75 & 13 & 0 & \\
\hline The K6 socre & $5.7 \pm 4.9$ & $3.6 \pm 3.7$ & $3.8 \pm 3.9$ & $0.0 \pm 0.0$ & 0.02 \\
\hline Cooperation with specialist & & & & & \\
\hline Number of subjects & 13 & 98 & 104 & 41 & \\
\hline The K6 socre & $3.2 \pm 2.4$ & $4.6 \pm 4.5$ & $5.3 \pm 4.8$ & $5.6 \pm 4.8$ & 0.239 \\
\hline
\end{tabular}

${ }^{* *} p<0.01$ by ANOVA (Tukey).

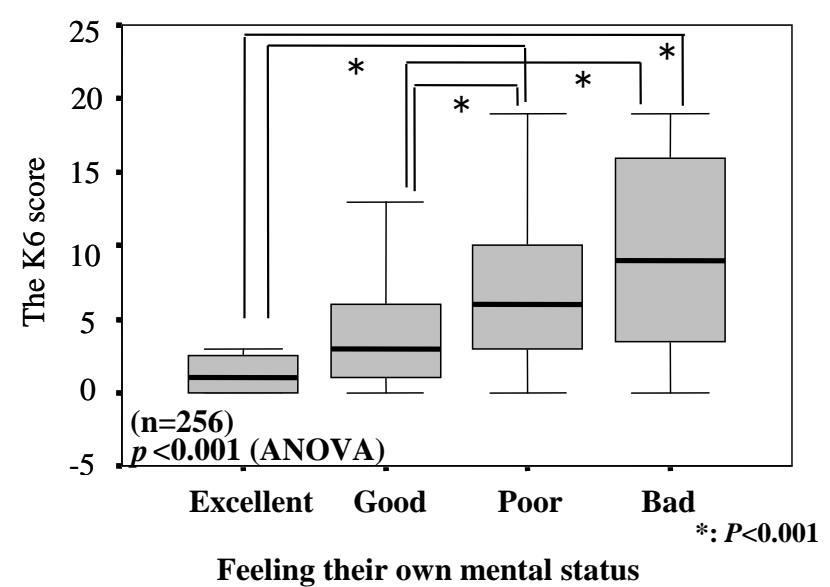

Figure 3. Relationship between the K6 score and feelings of their own mental status in public health nurse in Kagawa prefecture, Japan.

K6 in public health nurses in Kagawa prefecture, Japan.

According to the link between psychological distress and nurses in hospitals, Katayama has reported that the aspects of "suppressed expression”, “deep adjustment”, and "surface adjustment" of emotional labor seem to be the major occupational stressors for nurses [3]. Tei-Tominaga et al. showed that they obtained 301 newly graduated nurses and found that psychological distress was important predictor of intentions to leave in a longitudinal analysis [4]. Ishihara et al. also reported that the levels of urinaly 8-hydroxydeosyguanosine (8-OHdG), which reflects oxidative DNA injuries, in subjects with shift work was higher than that in subjects with part time work [6]. Munakata et al. also showed that psychological disturbances after night work were associated with altered cardiovascular and endocrine responses in healthy nurses [7]. In this study, we evaluated the psychological distress using the K6 in public health nurses in Kagawa prefecture, Japan. Consciousness of suicide and anxiety of preventing suicide in their daily work might be associated with psychological distress in public health nurses in Kagawa prefecture, Japan. Therefore, it seems reasonable to suggest that simply supporting in public health nurses in mental health and suicide is required in Kagawa prefecture, Japan.

In some literatures, psychological distress by using the K6 has been reported. Kuriyama et al. reported that they evaluated psychological distress of 43,716 communitydwelling people aged 40 years or older living in Japan [12]. Inoue et al. showed that part time workers in men and temporary/contract workers in women were associated with poor mental health in a total of 9461 men and 7777 women employees. No clear relation in mental health was noted in company size or occupation [13]. Subjective social status seems to be a stronger predictor of psychological distress among both men and women in the Japanese community than traditional socioeconomic status [14]. However, there was no report of psychological distress of public health nurse in Japan. In this study, by using the K6, we evaluated the psychological distress of public health nurse in Kagawa prefecture, Japan. A total of 15 public health nurses (5.9\%) was defined as psychological distress. The rate of psychological distress was similar to that previous report [12]. However, some factors in their daily work might be associated with their psychological distress. This reference and pilot data obtained from this study will be useful for preventing psychological distress in public health nurses in Japan.

Potential limitations still remain in this study. First, our study was a cross sectional and not a longitudinal study. Second, 256 public health nurses in our study voluntarily underwent measurement: they were therefore more likely to be health-conscious compared with the average public health nurses. To show this, further prospective studies are needed in the Japanese.

\section{REFERENCES}

[1] Prince, M., Patel, V., Saxena, S., Maj, M., Maselko, J., Phillips, M.R. and Rahman, A. (2007) No health without 
mental health. Lancet, 370, 859-877. doi:10.1016/S0140-6736(07)61238-0

[2] Cabinet Office, Government of Japan (2010). http://www8.cao.go.jp/jisatsutaisaku/whitepaper/w-2010/ pdf/gaiyou/

[3] Katayama, H. (2010) Relationship between emotional labor and job-related stress among hospital nurses. Nippon Eiseigaku Zasshi, 65, 524-529. doi:10.1265/jjh.65.524

[4] Tei-Tominaga, M. and Miki, A. (2010) A longitudinal study of factors associated with intentions to leave among newly graduated nurses in eight advance treatment hospitals in Japan. Industrial Health, 48, 305-316. doi:10.2486/indhealth.48.305

[5] Kawano, Y. (2008) Association of job-related stress factors with psychological and somatic symptoms among Japanese hospital nurses: Effect of departmental environment in acute care hospitals. Journal of Occupational Health, 50, 79-85. doi:10.1539/joh.50.79

[6] Ishihara, I., Nakano, M., Ikushima, M., Hara, Y., Yoshimine, T., Haraga, M., Nakatani, J., Kawamoto, R. and Kasai, H. (2008) Effect of work conditions and work environments on the formation of 8-OHdG in nurses and non-nurse female workers. Journal of UOEH, 30, 293308.

[7] Munakata, M., Ishi, S., Nunokawa, T., Saito, Y., Ito, N., Fukudo, S. and Yoshinaga, K. (2001) Influence of night shift work on psychologic state and cardiovascular and neuroendocrine responses in healthy nurses. Hypertension Research, 24, 25-31. doi:10.1291/hypres.24.25

[8] Kessler, R.C., Andrews, G., Colpe, L.J., Hiripi, E., Mroczek, D.K., Normand, S.L., Walters, E.E. and Zaslavsky, A.M. (2002) Short screening scales to monitor population prevalences and trends in non-specific psychological distress. Psychological Medicine, 32, 959-976. doi:10.1017/S0033291702006074

[9] Furukawa, T.A., Kessler, R.C., Slade, T. and Andrews, G.
(2003) The performance of the K6 and K10 screening scales for psychological distress in the Australian National Survey of Mental Health and Well-Bing. Psychological Medicine, 33, 357-362. doi:10.1017/S0033291702006700

[10] Kessler, R.C., Barker, P.R., Colpe, L.J., Epstein, J.F., Gfroerer, J.C., Hiripi, E., Howes, M.J., Normand, S.L., Manderscheid, R.W., Walters, E.E. and Zaslavsky, A.M. (2003) Screening for serious mental illness in the general population. Archives of General Psychiatry, 60, 184-189. doi:10.1001/archpsyc.60.2.184

[11] Furukawa, T.A., Kawakami, N., Saitoh, M., Ono, Y., Nakane, Y., Nakamura, Y., Tachimori, H., Iwata, N., Uda, H., Nakane, H., Watanabe, M., Naganuma, Y., Hata, Y., Kobayashi, M., Miyake, Y., Takeshima, T. and Kikkawa, T. (2008) The performance of the Japanese version of the K6 and K10 in the World Mental Health Survey Japan. International Journal of Methods in Psychiatric Research, 17, 152-158. doi:10.1002/mpr.257

[12] Kuriyama, S., Nakaya, N., Ohmori-Matsuda, K., Shimazu, T., Kikuchi, N., Kakizaki, M., Sone, T., Sato, F., Nagai, M., Sugawara, Y., Akhter, M., Higashiguchi, M., Fukuchi, N., Takahashi, H., Hozawa, A. and Tsuji, I. (2009) Factors associated with psychological distress in a community dwelling Japanese population: The Ohsaki Cohort 2006 Study. Journal of Epidemiology, 19, 294-302. doi:10.2188/jea.JE20080076

[13] Inoue, A., Kawakami, N., Tsuchiya, M., Sakurai, K. and Hashimoto, H. (2010) Association of occupation, employment contract, and company size with mental health in a national representative sample of employees in Japan. Journal of Occupational Health, 52, 227-240. doi:10.1539/joh.O10002

[14] Sakurai, K., Kawakami, N., Yamaoka, K., Ishikawa, H. and Hashimoto, H. (2010) The impact of subjective and objective social status on psychological distress among men and women in Japan. Social Science \& Medicine, 70, 1832-1839. doi:10.1016/j.socscimed.2010.01.019 